\title{
Investigation of optical short pulse generation using intensity modulation for LiDAR and free-space communications
}

\author{
FEI WANG ${ }^{1 *}$, WEN KANG ${ }^{2}$ \\ ${ }^{1}$ School of Physical Science and Technology, Southwest University, \\ Chongqing 400715, P.R. China \\ ${ }^{2}$ Institute of Electrical and Electronic Engineering, Chongqing University of Technology, \\ Chongqing 400054, P.R. China \\ *Corresponding author: wangf17@swu.edu.cn
}

\begin{abstract}
A simple optical pulse generation scheme for pulsed light detection and ranging (LiDAR) and free -space communications is proposed and experimentally and numerically demonstrated, in which continuous-wave light emitted by a distributed feedback-laser diode (DFB-LD) is modulated by a lithium-niobate Mach-Zehnder intensity modulator to generate optical short pulses, and an Er-doped fiber amplifier (EDFA) is used to boost transmitting light power. Possibilities of intensity modulators for high speed communications being used to generate optical short pulses for low speed pulsed LiDAR are investigated. The influences of bias voltage of intensity modulator and bit rate of modulation signal on the generated optical pulses are discussed. The pulse width obtained by using the return to zero signal with $33 \%$ duty cycle on bit rate of 2.5 and $5 \mathrm{Gbit} / \mathrm{s}$ is respectively 128.0 and $63.2 \mathrm{ps}$, and the corresponding nominal accuracy of pulsed LiDAR is respectively 19.2 and $9.5 \mathrm{~mm}$, and low repetition frequency required by pulsed LiDAR is achieved by coding to high -speed modulation signal. The system performance for free-space communications and pulsed LiDAR is evaluated, respectively. We believe that the proposed scheme is suitable for the integrated system of pulsed LiDAR and free-space communications.
\end{abstract}

Keywords: intensity modulator, optical pulse generator, pulsed LiDAR, free-space communications.

\section{Introduction}

Light detection and ranging (LiDAR) is a remote sensing method that uses a laser beam to measure target ranges. In general, LiDAR systems can be divided into two categories, namely, pulsed LiDAR and modulated continuous-wave (CW) LiDAR systems according to the modulation style of their light sources [1]. LiDAR has aroused considerable research interest in various areas such as autonomous vehicle [2], obstacle detection [ $\underline{3}$ ] and security [4] , and so on. Especially, research interest in automotive LiDAR is growing rapidly because of its clear accuracy and ability to detect a wide 
range of objects within a few hundred meters in all weather conditions and lighting conditions [ [5]. As core part of pulsed LiDAR system, performance of laser light source directly affects performance of LiDAR system. For pulsed LiDAR, repetition rate of light source determines detection range and sampling rate, and pulse width determines detection accuracy. Many methods have been proposed to generate optical short pulses, such as mode locking [] ], $Q$-switching [7]], electro-optic modulation [ [ $]$ ], pulse pumping [9], and so on. The compact structure of a mode-locked laser diode can produce a very short pulse and has relatively high repetition frequency, but oscillation wavelength and repetition frequency are difficult to be tuned in a large range, and mode-partition noise is serious. $Q$-switched fiber lasers are used to provide near-Gaussian pulse with high signal-to-noise ratio in nanosecond range, but some pulse parameters, such as, duration, repetition rate and pulse shape, cannot be adjusted independently. The method of modulating CW light by an electro-absorption modulator (EAM) is flexible and adjustable, especially suitable for photon integration system, but fiber-pigtailed EAM has great insertion loss and the cost is relatively high. Pulse width obtained by pulse pumping is related to many factors and not easy to be determined and controlled. Therefore, a compact optical short pulse source with tunable wavelength and repetition frequency is very attractive for pulsed LiDAR.

The intelligent driving assistance system (IDAS) is one of the most promising solutions for the high mortality rate of traffic accidents in the world today [10]. LiDAR is an indispensable core sensor component in IDAS. In addition, vehicle-to-vehicle (V2V) communications also play an important role in improving vehicle safety, reducing environmental pollution and saving energy by transmitting information between vehicles [11]. The LiDAR system integrates ranging and communications within only one set of hardware devices. For example, a three-dimensional imaging LiDAR system based on high speed pseudorandom modulation and photon counting was reported [12]. High-performance integrated optical phased arrays for LiDAR and free-space data communication were presented and demonstrated [13]. However, so far, relevant reports are still very few.

In this article, a compact and simple optical pulse generator for pulsed LiDAR and $\mathrm{V} 2 \mathrm{~V}$ free-space communications is proposed and experimentally and numerically demonstrated. The CW light emitted from a distributed feedback-laser diode (DFB-LD) is modulated by a lithium-niobate Mach-Zehnder intensity modulator (IM) to generate optical short pulse, and an Er-doped fiber amplifier (EDFA) is used to boost light transmitting power. However, the generated shortest optical pulse width is limited by the electric modulation pulse width generated by the employed low speed arbitrary waveform generator (AWG), even if a wideband linearly chirped fiber Bragg grating (LCFBG) is adopted to compress the generated light pulse, pulse compression still is very limited. So, the influences of bias voltage of IM and bit rate of modulation signal on the generated optical pulse are investigated. Since high-speed modulation results in narrow pulse generation, an intensity modulator for high speed communications is used to low 
speed pulsed LiDAR. The pulse width obtained by using the return to zero (RZ) signal with $33 \%$ duty cycle on bit rate of 2.5 and $5 \mathrm{Gbit} / \mathrm{s}$ is respectively 128.0 and $63.2 \mathrm{ps}$, and the corresponding nominal accuracy of pulsed LiDAR is respectively 19.2 and $9.5 \mathrm{~mm}$, and low repetition frequency required by pulsed LiDAR is achieved by coding to the modulation signal with a high bit rate. Since LiDAR and V2V communications are two indispensable functions of IDAS and unmanned vehicle, the integration of these two functions can effectively reduce size, weight, power consumption and cost of the system. We believe that the proposed scheme for optical short pulse generation has the potential to be applied to integrated system including pulsed LiDAR and V2V communications.

\section{Experimental principle and setup}

Configuration of the optical pulse generator is shown in Fig. 1, which consists of a CW laser with a 1-MHz line width, AWG (RIGOL DG1022U), lithium-niobate IM (CONQUER KG-AMOBOX) with a 10-GHz modulation bandwidth, and EDFA with small signal gain of $25 \mathrm{~dB}$. The $\mathrm{CW}$ emitted by the DFB-LD is modulated by the lithium-niobate MZM driven by RF signal generated by the AWG with a low repetition frequency, and the power of generated light pulse is amplified by the EDFA. To get a shorter pulse, a wideband LCFBG with a central wavelength of $1551.10 \mathrm{~nm}$ and bandwidth of $16.16 \mathrm{~nm}$, as a group delay dispersion medium with a ripple delay, is used to compress the generated light pulse via an optical circulator; moreover, it is also possible to suppress timing jitters during pulse compression [14]. The generated light pulse is monitored by using a photoelectric detector $\left(u^{2} t\right.$, XPDV2120R) with a $50-\mathrm{GHz}$ bandwidth and an oscilloscope (RIGOL, DS7054).

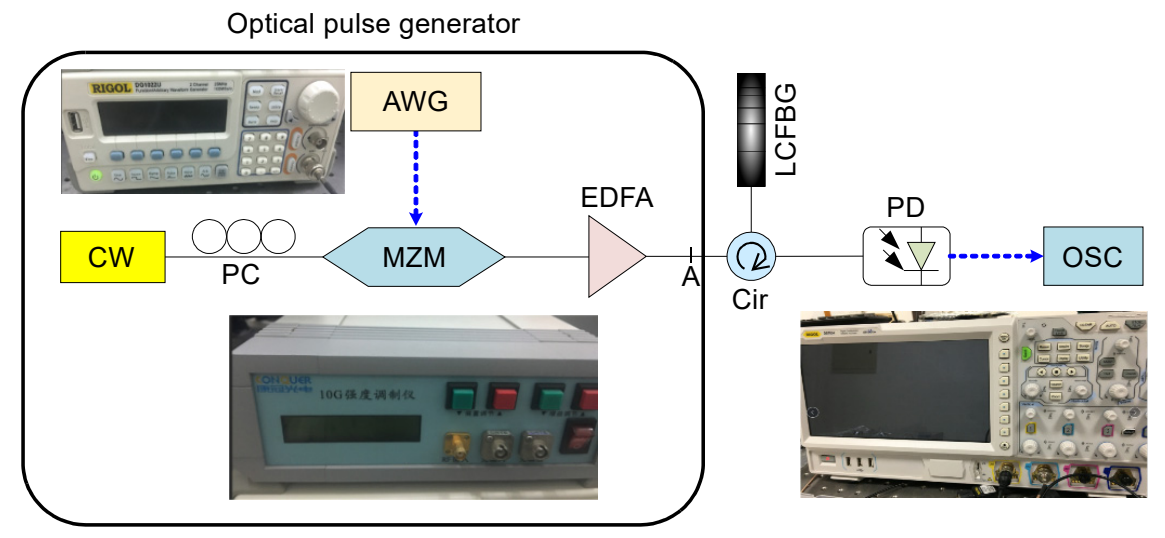

Fig. 1. Configuration of the optical pulse generator. PC: polarization controller, MZM: Mach-Zehnder modulator, AWG: arbitrary waveform generator, EDFA: Er-doped fiber amplifier, Cir: circulator, LCFBG: linearly chirped fiber Bragg grating, PD: photodetector, OSC: oscilloscope. 
The principle of the optical pulse generator based on intensity modulation is described as follows. The electrical field of CW light from the DFB-LD is expressed as

$$
E(t)=E_{0} \exp \left(-j \omega_{0} t\right),
$$

here, $E_{0}$ is the amplitude of the electrical field and $\omega_{0}$ is the angular frequency. The transmission function of the Mach-Zehnder intensity modulator can be expressed as

$$
H=\frac{I_{\text {out }}}{I_{\text {in }}}=\left|\frac{E_{\text {out }}}{E_{\text {in }}}\right|^{2}=\frac{1}{2}+\frac{1}{2} \sin \left\{\frac{\pi}{V_{\pi}}\left[V_{\mathrm{m}}(t)-V_{\mathrm{DC}}\right]\right\}
$$

where $I_{\text {out }}$ and $I_{\text {in }}$ are respectively the output and input light intensity, $E_{\text {out }}$ and $E_{\text {in }}$ are respectively the electric field component of the output and input light, $V_{\mathrm{m}}(t)$ is the electrical modulation signal, $V_{\mathrm{DC}}$ is $\mathrm{DC}$ bias of the modulator. If the repetition rate of the modulation signal is $f_{\mathrm{m}}$, the sinusoidal electrical modulation signal can be expressed as

$$
V_{\mathrm{m}}(t)=V_{0} \sin \left(\pi f_{\mathrm{m}} t\right)
$$

where $V_{0}$ is the amplitude of the electrical modulation signal. When $V_{\mathrm{DC}}=2 n \pi-\pi / 2$, the output light intensity after being modulated is

$$
\begin{aligned}
I_{\text {out }}(t) & =I_{\text {in }} \frac{1}{2}\left\{1+\cos \left[\frac{\pi}{V_{\pi}} V_{0} \sin \left(\pi f_{\mathrm{m}} t\right)\right]\right\} \\
& =I_{\text {in }} \cos ^{2}\left[\frac{\pi}{2 V_{\pi}} V_{0} \sin \left(\pi f_{\mathrm{m}} t\right)\right]
\end{aligned}
$$

where $V_{\pi}$ is the half-wave voltage of the modulator. The system performance of the pulsed LiDAR is closely related to the employed optical pulse. For example, the furthest distance $R_{\max }$ that can be detected by the pulsed LiDAR can be expressed as

$$
R_{\max }=\frac{1}{2} c T=\frac{c}{2 f}
$$

here, $c$ is the speed of light, $T$ is the pulse period, and $f$ is the repetition frequency. The detection distance of the LiDAR is inversely proportional to the repetition frequency of the generated pulse. The accuracy $\Delta R$ of the pulsed LiDAR can be expressed as

$$
\Delta R=\frac{c t_{\mathrm{p}}}{2}
$$

here, $t_{\mathrm{p}}$ is the pulse width. So, the accuracy of the LiDAR is proportional to the pulse width, namely, the shorter pulse width means the higher ranging accuracy. Therefore, how to obtain the shortest optical pulse width and appropriate repetition rate for the pulsed LiDAR will be discussed. 


\section{Results and discussion}

The electric modulation pulse trains generated by the AWG at different repetition frequencies are shown in Fig. 2. The repetition rate of the electric modulation pulse trains generated by the AWG is 10,50,100,200, 300 and $400 \mathrm{kHz}$, respectively. All electric

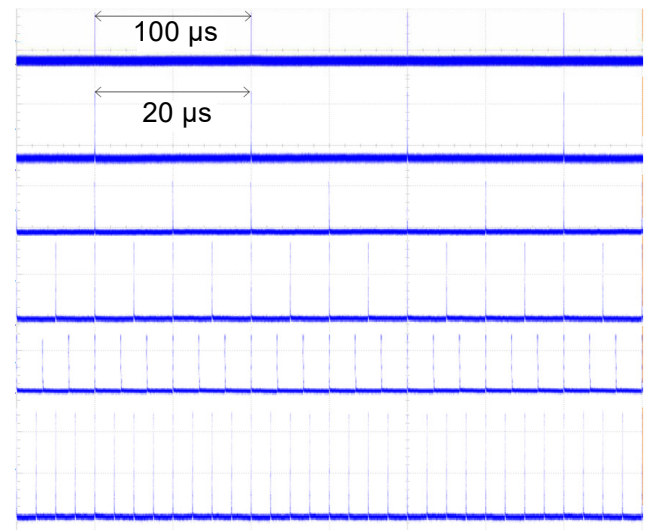

Fig. 2. The electric modulation pulse trains with different repetition frequency.
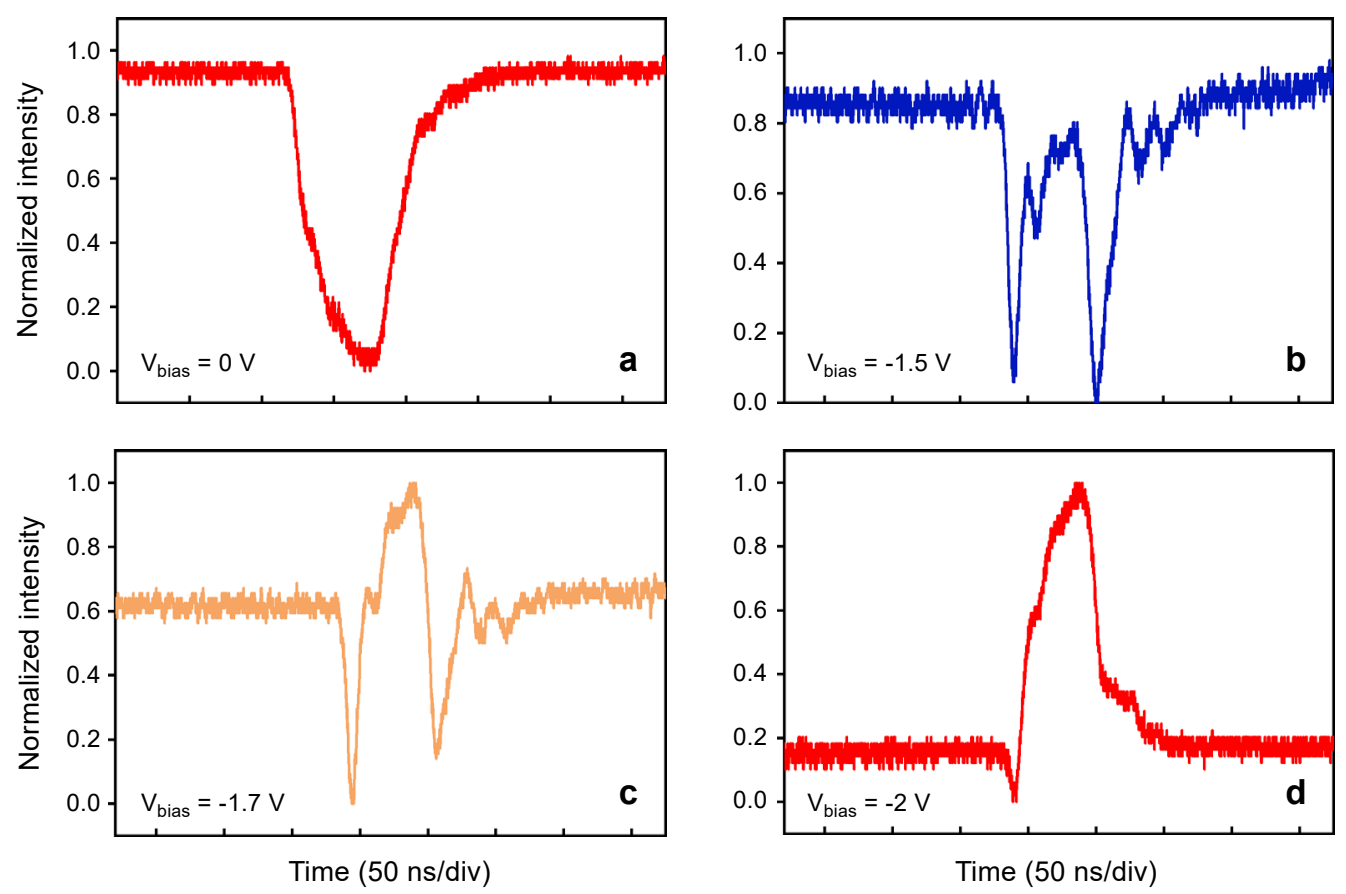

Fig. 3. Waveform evolution of the optical pulse generated by intensity modulation with low repetition frequency on different bias voltage of the intensity modulator (see text for explanation). 
modulation pulse trains have the same amplitude, namely, high level is $1.5 \mathrm{~V}$ and low level is $0 \mathrm{~V}$.

Figure 3 shows the waveform evolution of the optical pulse generated by the intensity modulation with a low repetition frequency when the bias voltage of the intensity modulator is adjusted. The repetition frequency of the modulation signal is kept on $10 \mathrm{kHz}$, high level and low level of the modulation signal is respectively kept in 1.5 and $0 \mathrm{~V}$. When the bias voltage of the intensity modulator is $0 \mathrm{~V}$, a dark pulse is obtained, as shown in Fig. $3 \mathbf{a}$. From 0 to $-1.5 \mathrm{~V}$, the center part of the dark pulse reverses and reaches the DC level when the bias voltage is $-1.5 \mathrm{~V}$, as shown in Fig. $3 \mathbf{b}$. As the bias voltage keeps going down, the inverted dark pulse goes up to the DC level and becomes a positive pulse. When the bias voltage reaches $-1.7 \mathrm{~V}$, the pulse with the minimum pulse width is obtained. However, the extinction ratio of the obtained pulse is very low, as shown in Fig. 3c. When the bias voltage reaches $-2 \mathrm{~V}$, a positive pulse with a narrower pulse width and a higher extinction ratio is obtained, as shown in Fig. 3d. Similar evolution of a pulse shape is also observed in the positive bias voltage region of an intensity modulator.

The CW emitted by the DFB-LD is modulated in the MZM driven by the electric modulation pulse trains. The power of the generated light pulse is amplified by an EDFA, and then the pulse is compressed by a wideband LCFBG. Figure 4 shows the pulse width of the electric modulation pulses, generated uncompressed optical pulses (point A in Fig. 1) and compressed optical pulses at different repetition frequencies. When the repetition rate is $10 \mathrm{kHz}$, the generated shortest electric-modulation pulse width is $49.5 \mathrm{~ns}$, the generated uncompressed pulse is $49.0 \mathrm{~ns}$, and the compressed pulse width after through LCFBG is $46.3 \mathrm{~ns}$. When the repetition rate is $50 \mathrm{kHz}$, the generated shortest electric-modulation pulse width is $22.3 \mathrm{~ns}$, the generated uncompressed pulse is $20.4 \mathrm{~ns}$, and the compressed pulse width after through LCFBG is $17.9 \mathrm{~ns}$. When the repetition

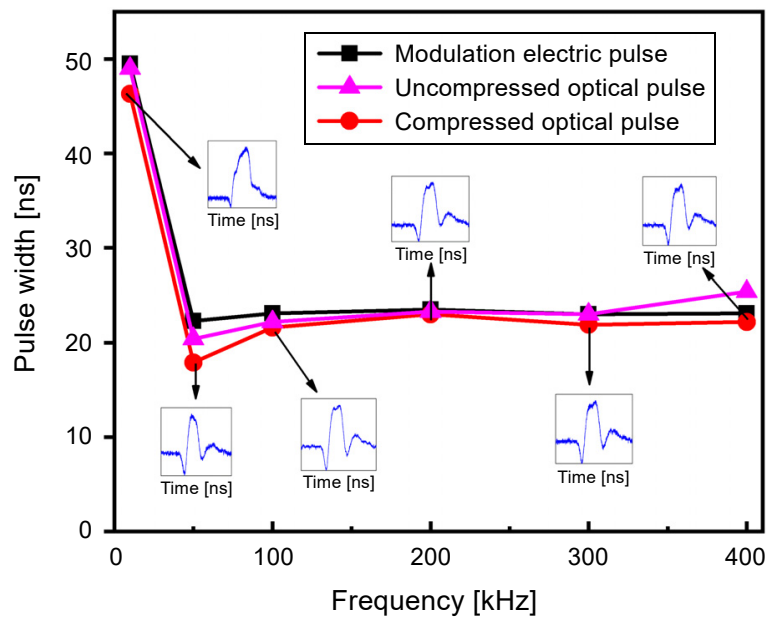

Fig. 4. Pulse width of the electric modulation pulses, generated uncompressed optical pulses and compressed optical pulses at different repetition frequencies. 
rate is $100 \mathrm{kHz}$, the generated shortest modulation-electric pulse width is $23.1 \mathrm{~ns}$, the generated uncompressed pulse is $22.2 \mathrm{~ns}$, and the compressed pulse width after through LCFBG is $21.6 \mathrm{~ns}$. When the repetition frequency of the AWG is set to 200, 300 and $400 \mathrm{kHz}$, the generated shortest electrical pulse width is $23.5,23.0,23.1 \mathrm{~ns}$, the generated uncompressed optical pulse width is $23.3,23.0,25.4 \mathrm{~ns}$, and the compressed optical pulse width after passing LCFBG is $23.0,21.9,22.2 \mathrm{~ns}$, respectively. The insets show the pulse waveform of the compressed pulses at different repetition frequencies. Although the repetition rate of the electric pulse from the employed AWG can be tuned to meet the needs of LiDAR detection, the generated optical pulse width is limited by the shortest electric pulse width generated by the AWG. Even if the pulse compression technology which is widely used in optical communication is adopted to compress the generated light pulse, the compression effect is still very poor. This is because, when LCFBG is used to a pulse compressor, the pulse should first pass through a normal dispersion fiber, and through the joint action of self-phase modulation (SPM) and group velocity dispersion (GVD), an approximate linear positive chirp across the whole pulse is generated. Then, the LCFBG provides the anomalous GVD needed to compress the positive chirped pulse. However, this inevitably leads to an increase in the complexity of the system. In addition, the pulse width should be generally in ps magnitude, and the compression factor of LCFBG is also limited.

Since LiDAR and V2V communications are two indispensable functions for IDAS and unmanned vehicles, the integration of these two functions can effectively reduce the size, weight, power consumption and cost of system. V2V communications are expected to be high-speed, and high-speed modulation results in narrow pulse generation, and the generated narrow pulse provides high accuracy for pulsed LiDAR. As for the low repetition frequency required by the pulsed LiDAR, it can be realized by coding to a high-speed modulation signal. For example, a $2.5-\mathrm{Gb} / \mathrm{s}$ electric modulation signal is set to " 1 " for every 5000 bits, so, a signal with a repetition frequency of $500 \mathrm{kHz}$ can be obtained. Therefore, optical short pulses with low repetition frequency can be obtained by using an intensity modulator with a high modulation bandwidth. Moreover, functional integration of the pulsed LiDAR and high-speed free-space communications are also feasible in principle. To accommodate the hybrid system of the pulsed LiDAR and free-space communications, the low-speed AWG was replaced by a high-speed signal generator. However, due to the limitation of experimental conditions, we will study the system characteristics by numerical simulation. Pulse width generated by the electric modulation signal with different modulation formats and bit rates, and the corresponding nominal accuracy of the pulsed LiDAR are shown in Fig. 5. Pulse widths generated by the electric modulation signal with different modulation formats and bit rates are shown in Fig. 5a. For all modulation formats employed, including the non-return to zero (NRZ) modulation format and the return to zero (RZ) modulation format with different duty ratio, as a bit rate of the signals increases, the generated pulse width decreases gradually. The pulse width generated by RZ modulation format is narrower than that generated by NRZ modulation format. To further reduce the pulse width, RZ signals with different duty ratio are used, which are set to 66,50 , and $33 \%$, respectively. 


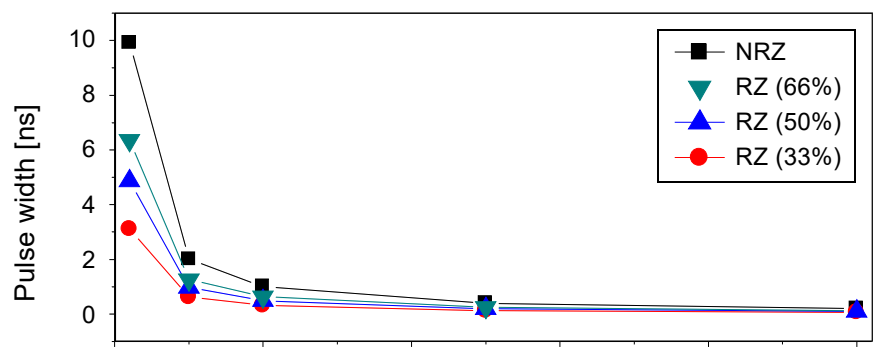

a

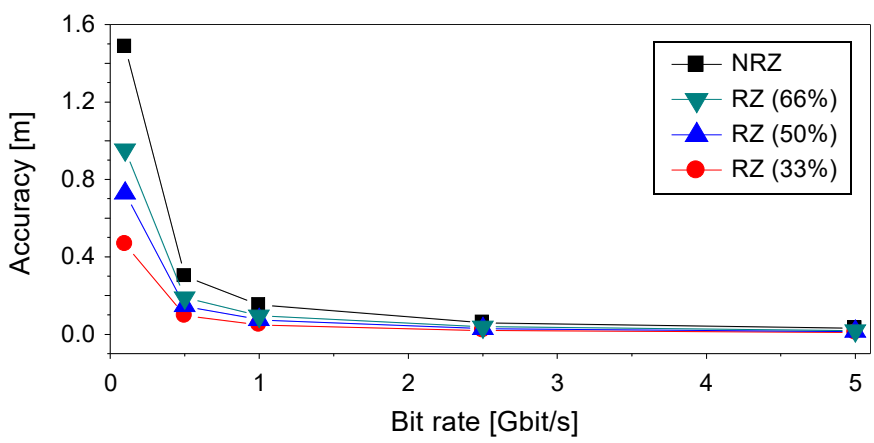

b

Fig. 5. Pulse widths generated by the electric modulation signals with different modulation formats and repetition frequencies (a), and the corresponding nominal accuracy of the pulsed LiDAR (b).

By contrast, the narrowest pulse width is obtained by using the RZ signal with $33 \%$ duty cycle. The corresponding nominal accuracy of the pulsed LiDAR to the pulses generated is shown in Fig. 5b. According to Eq. (5), the detection accuracy of the pulsed LiDAR is proportional to the pulse width, and the trend of nominal accuracy of the pulsed LiDAR changes with a bit rate is similar to that of the pulse width. The pulse width obtained by using the RZ signal with $33 \%$ duty cycle on a bit rate of 2.5 and $5 \mathrm{Gbit} / \mathrm{s}$ is respectively 128.0 and $63.2 \mathrm{ps}$, and the corresponding nominal accuracy of the pulsed LiDAR is respectively 19.2 and $9.5 \mathrm{~mm}$.

Since the proposed scheme is designed for the integrated system of free-space communications and pulsed LiDAR, the communication performance of the integrated system is first evaluated. A free-space communications link is established, and typical system parameters are as follows. The wavelength of the laser is set at $1550 \mathrm{~nm}$, the bit rate of the data is $2.5 \mathrm{Gbit} / \mathrm{s}$, the distance of free-space transmission link is $1 \mathrm{~km}$, the attenuation of beam divergence is $70 \mathrm{~dB} / \mathrm{km}$, the aperture diameter of the transmitter and receiver is respectively 5 and $20 \mathrm{~cm}$, and the responsibility of the avalanche photodiode (APD) is $1 \mathrm{~A} / \mathrm{W}$. BER measurement results of 1-km free-space communications link for different modulation formats are shown in Fig. 6. The results show that the power penalty induced by NRZ and RZ with a duty ratio of $66,50,33 \%$ is respectively 1.2, 1.6, and $2.9 \mathrm{~dB}$. If the pulsed LiDAR is not considered, NRZ modulation format should be the first choice for free-space communications. In order to combine the pulsed LiDAR and free-space communications, choosing the modulation format 


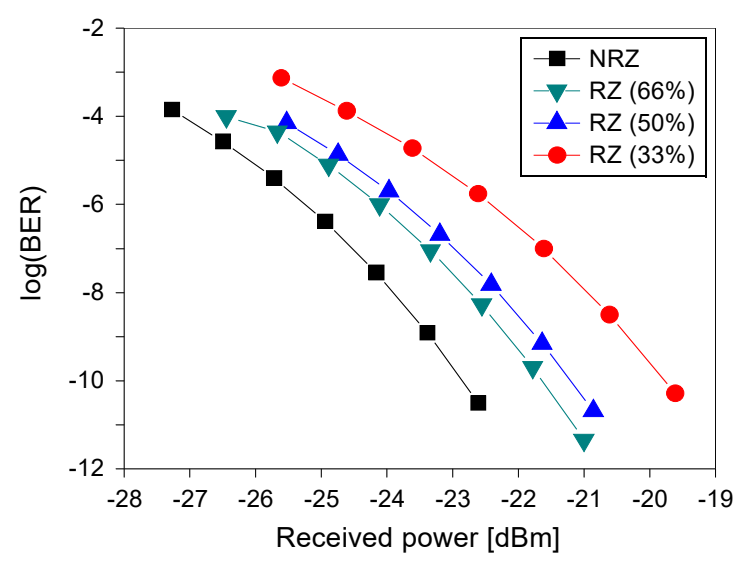

Fig. 6. BER measurements of 1-km free-space communication link for different modulation formats.

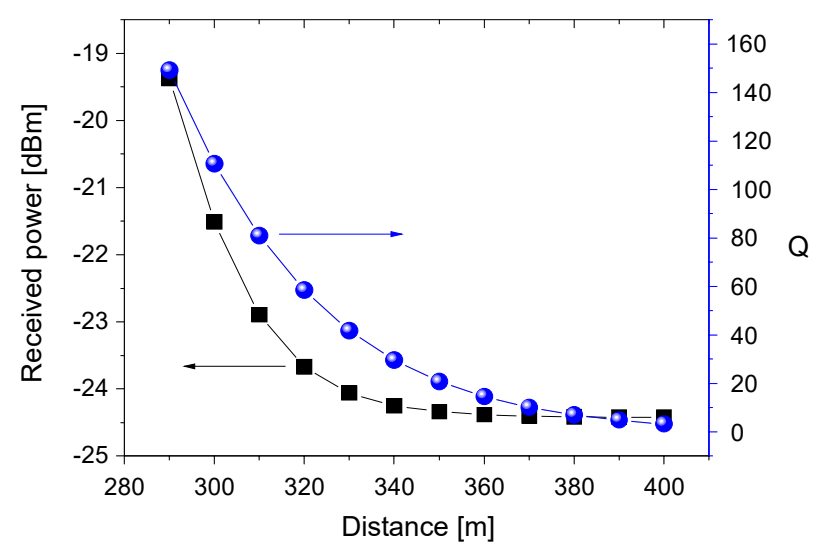

Fig. 7. Received light power and $Q$ values of the return optical pulse measured at different distances.

should consider both the bit error rate of communications and the accuracy of the pulsed LiDAR. Therefore, the pulsed LiDAR characteristics of the system are also measured. In order to improve the accuracy of ranging, the RZ modulation format with the minimum duty ratio of $33 \%$ is chosen to test. The received light power and $Q$ values of the return optical pulse measured at different distances are shown in Fig. 7. As the detection range increases, the received light power and $Q$ value of the return optical pulse decrease gradually. In order to increase the range of ranging, the transmitting power, the gain of preamplifier and the photodetector should be increased.

\section{Conclusion}

We have proposed and experimentally demonstrated a compact and simple optical pulse generator, which could be applied to integrated system of pulsed LiDAR and free-space 
communications. The intensity modulator for high speed communications can generate low speed short pulse for pulsed LiDAR applications with high accuracy, by coding to a high-speed modulation signal. The system performances for free-space communications and pulsed LiDAR have been evaluated, respectively. In the following work, the time division multiplex (TDM) or other multiplex mechanisms for the integrated system of free-space communications and pulsed LiDAR still need further be researched.

Acknowledgements - This work was partly supported by the Central Universities of China under Grant SWU020001, the National Natural Science Foundation of China (61575034) and the recruitment program of global experts (WQ20165000357).

\section{References}

[1] Tsai C.-M., LiU Y.-C., Anti-interference single-photon LiDAR using stochastic pulse position modulation, Optics Letters 45(2), 2020, pp. 439-442, DOI: 10.1364/OL.384894.

[2] Hata A.Y., Wolf D.F., Feature detection for vehicle localization in urban environments using a multilayer LIDAR, IEEE Transactions on Intelligent Transportation Systems 17(2), 2016, pp. 420-429, DOI: 10.1109/TITS.2015.2477817.

[3] Ramasamy S., Sabatini R., Gardi A., Liu J., LIDAR obstacle warning and avoidance system for unmanned aerial vehicle sense-and-avoid, Aerospace Science and Technology 55, 2016, pp. 344-358, DOI: $10.1016 /$ j.ast.2016.05.020.

[4] Schwarz B., LIDAR: Mapping the world in 3D, Nature Photonics 4(7), 2010, pp. 429-430, DOI: 10.1038/nphoton.2010.148.

[5] Shi J.-W., Guo J.-I., Kagami M., Suni P., Ziemann O., Photonic technologies for autonomous cars: feature introduction, Optics Express 27(5), 2019, pp. 7627-7628, DOI: 10.1364/OE.27.007627.

[6] Zhang Z., CAI Y., WANG J., WAN H., Zhang L., Switchable dual-wavelength cylindrical vector beam generation from a passively mode-locked fiber laser based on carbon nanotubes, IEEE Journal of Selected Topics in Quantum Electronics 24(3), 2018, article 1100906, DOI: 10.1109/JSTQE.2017. 2761126.

[7] Salam S., Al-Masoodi A.H.H., Wang P., Harun S.W., Hybrid organic small molecules as a saturable absorber for passive Q-switching in erbium-doped fiber laser, OSA Continuum 3(2), 2020, pp. 177-185, DOI: $10.1364 /$ OSAC.379189.

[8] Chou H.-F., Chiu Y.-J., Bowers J.E., Standing-wave enhanced electroabsorption modulator for 40-GHz optical pulse generation, IEEE Photonics Technology Letters 15(2), 2003, pp. 215-217, DOI: $10.1109 /$ lpt.2002.806850.

[9] Gorjan M., PetKovšek R., MarinČEk M., Čopič M., High-power pulsed diode-pumped Er:ZBLAN fiber laser, Optics Letters 36(10), 2011, pp. 1923-1925, DOI: 10.1364/OL.36.001923.

[10] Cheng H., Zheng N., Zhang X., Qin J., vAn DE Wetering H., Interactive road situation analysis for driver assistance and safety warning systems: framework and algorithms, IEEE Transactions on Intelligent Transportation Systems 8(1), 2007, pp. 157-167, DOI: 10.1109/TITS.2006.890073.

[11] Thunberg J., Lyamin N., SJöberg K., Vinel A., Vehicle-to-vehicle communications for platooning: safety analysis, IEEE Networking Letters 1(4), 2019, pp. 168-172, DOI: 10.1109/LNET.2019.2929026.

[12] Zhang Y., He Y., Yang F., Luo Y., Chen W., Three-dimensional imaging lidar system based on high speed pseudorandom modulation and photon counting, Chinese Optics Letters 14(11), 2016, article 111101, DOI: 10.3788/COL201614.111101.

[13] Poulton C.V., Byrd M.J., Russo P., Timurdogan E., Khandaker M., Vermeulen D., Watts M.R., Long-range LiDAR and free-space data communication with high-performance optical phased ar- 
rays, IEEE Journal of Selected Topics in Quantum Electronics 25(5), 2019, article 7700108, DOI: 10.1109/JSTQE.2019.2908555.

[14] Williams J.A.R., Bennion I., Zhang L., The compression of optical pulses using self-phase-modulation and linearly chirped Bragg-gratings in fibers, IEEE Photonics Technology Letters 7(5), 1995, pp. 491-493, DOI: $\underline{10.1109 / 68.384520 .}$.

Received February 8, 2020

in revised form April 4, 2020 